\title{
An approach to polymer degradation through microbes
}

\author{
Uttiya Dey ${ }^{1}$, Naba Kumar Mondal ${ }^{2 *}$, Kousik Das ${ }^{3}$ and Shampa Dutta ${ }^{4}$ \\ ${ }^{1,3}$ (Research scholar, Department of Environmental Science, The University of Burdwan, West Bengal, India) \\ ${ }^{2,4}$ (Faculty, Department of Environmental Science, The University of Burdwan, West Bengal, India)
}

\begin{abstract}
Inertness and indiscriminate uses as well as growing water and land pollution problems have lead to concern about plastics. Present paper investigates the possibility of plastic degradation by microbes isolated from forest soil and automobile washout sludge. The in-vivo degradation was studied by litter bag experiment by taking $1 \mathrm{~g}$ of each plastic and buried under forest soil and automobile wash-out sludge at a depth of $15 \mathrm{~cm}$ from the surface during the month of September to February, 20102011. An in-vitro experiment was started after collecting the plastic samples from the litter bag experiment and the microbes were isolated from the surface of the plastic. Then the isolated microbes inoculated in the Burk's medium without carbon source in two sets, one with plastic and the other with polyethylene glycol (PEG). Result showed that no variety of plastic comfortable degraded under burial condition during six months. But interesting result was recorded from Scanning Electron Microscopy (SEM) study. The preliminary screening of biodegradation capability was done by Fourier Transform Infra Red (FTIR) Spectroscopy for surface changes. Again, degradation of PEG by microbes clearly indicates their existence in the said medium. Therefore it can be speculated that microbes has enough potential to degrade plastic with due course of time.
\end{abstract}

$\underline{\text { Keywords: Biodegradation, Plastics, Polyethylene glycol, Scanning electron microscope, Soil microbes. }}$

\section{INTRODUCTION}

Plastic is the most useful synthetic 'manmade' substance, made up of elements extracted from the fossil fuel resources. It has made possible most of the industrial and technological revolutions of the 19th and 20th centuries. During the past 30 years plastic materials have been used widely in food, clothing, shelter, transportation, construction, medical and leisure industries because they are lightweight, low cost, extremely durable and relatively unbreakable [1]. A very general estimate of worldwide plastic waste generation is annually about 57 million tons [2]. They do not break down in the environment easily because they are resistant to microbial attack, due to their excessive molecular mass, high number of aromatic rings, unusual bonds, or halogen substitutions [3]. As a result they remain in the environment for a very long time without any deterioration and the large-scale accumulation of waste plastics in the biosphere has given rise to the problem of severe environmental pollution [4]. These problems have made plastic waste a major focus in the management of solid waste.

Polymers are a broad class of materials which are made from repeating units of smaller molecules called monomers. Polymers can be natural in origin, such as the lignin of tree branches, the starches of homemade bread, or the chitin of lobster shells. Other polymers are called synthetic, because they are made by humans from naturally-occurring materials. Most polymers are too large to pass through cellular membranes, so they must first be depolymerized to small monomers before they can be absorbed and biodegraded within microbial cells. The initial breakdown of a polymer can result from a variety of physical, chemical, and biological forces [5]. There is considerable research on the development of biodegradable plastics as well as on the degradation of existing plastics using microorganisms. Since microorganisms are capable of degrading most of the organic and inorganic materials, including lignin, starch, cellulose, and hemicelluloses [6], there is lot of interest in the microbial degradation of plastic and polythene waste material. Kambe et al. in 1995 [7], isolated and characterized a bacterium from soil which utilizes polyester polyurethane as a sole carbon and nitrogen source. Recent research has revealed that the high diversity of the microorganisms in the mangrove soil is capable of degrading the plastics, although at a slower rate [8]. The growth of many fungi can also cause small-scale swelling and bursting, as the fungi penetrate the polymer solids [9]. Webb et al. in 2000 [10], studied the fungal colonization and biodegradation of plasticized polyvinyl chloride in in-situ and ex situ conditions. Microorganisms secrete a variety of enzymes into the soil water, which begin the breakdown of the polymers. Two types of enzymes are involved in the process, namely intracellular and extracellular depolymerases. Exoenzymes from the microorganisms first breakdown the complex polymers giving short chains or monomers that are small enough to permeate through the cell walls to be utilized as carbon and energy sources. The process is known as depolymerization. When the end product is carbon dioxide, water or methane, then the process is known as mineralization [11]. 
An attempt in this paper has been made to study the natural degradation of commonly used plastic under forest soil and automobile wash-out sludge and to isolate potential microorganism from forest soil and automobile wash-out sludge that might be able to degrade polyethylene glycol and commonly used plastic material.

\section{MATERIAL AND METHOD}

Nine types of commonly used plastic cups and plastic carry bags were used throughout the study. The samples were cut into small pieces of about $0.2-0.5 \mathrm{~cm}$ in diameter.

2.1 Degradation of plastic under natural condition: $1 \mathrm{~g}$ of each sample was taken in different litter bags and they were buried at a depth of about $5 \mathrm{~cm}$ under forest soil and automobile wash-out sludge for six months (September 2010 to February 2011). The samples were collected after six months, washed thoroughly with distilled water, Sun-dried and finally weighed for the final weight.

2.2 Isolation of microorganisms: Sample from soil and automobile wash-out sludge was taken to the laboratory and microbes from these samples were isolated by pour-plate method.

2.3 Inoculation of poly ethylene glycol and plastic with microbes: In the first set of experiment, in two conical flask, $20 \mathrm{ml}$ of synthetic medium was prepared in each conical flask without carbon source i.e., sucrose and $0.4 \mathrm{ml}$ of PEG was added as only carbon incubated at $27^{\circ} \mathrm{c}$ for 30 days. At the same time another set of experiment was formulated in which the only carbon source in the synthetic medium were different types of plastic used in the experiment rather than sucrose or PEG.

2.4 Viability test: After 30 days of incubation viability test was done by pour plate method to examine the living existence of the microbes.

2.5 Scanning electron micrograph: After 45 days of incubation all the plastic samples were taken out of the medium, dried and sent for scanning electron micrograph (SEM) to find if there is any deformity on the surface of the plastic.

\section{Result}

No neat change (weight, color, or physical nature) was recorded after incubation for six months under forest soil (Table-1). On the other hand, the result of automobile wash-out sludge experiment revealed that there was no change of any plastic except the biscuit wrapper where only $9 \%$ weight change was recorded along with slight color change (Table-2).

Table-1: Change of plastic during burial under forest soil.

\begin{tabular}{|c|l|l|l|l|l|l|}
\hline \multirow{2}{*}{ Sample } & \multicolumn{2}{|l|}{ Weight $(\mathrm{g})$} & Color of sample & \multicolumn{2}{l|}{ Form of sample } \\
\cline { 2 - 7 } & Initial & Final & Initial & Final & Initial & Final \\
\hline 1. & 1 & 1 & Transparent & Transparent & Chips & Chips \\
\hline 2. & 1 & 1 & Black & Black & Chips & Chips \\
\hline 3. & 1 & 1 & Pink & Pink & Chips & Chips \\
\hline 4. & 1 & 1 & White & White & Chips & Chips \\
\hline 5. & 1 & 1 & Orange & Orange & Chips & Chips \\
\hline 6. & 1 & 1 & Yellow & Yellow & Chips & Chips \\
\hline 7. & 1 & 1 & White & White & Chips & Chips \\
\hline 8. & 1 & 1 & Blue & Blue & Chips & Chips \\
\hline 9. & 1 & 1 & Blue & Blue & Chips & Chips \\
\hline
\end{tabular}


Table-2: Change of plastic during burial under automobile wash-out sludge.

\begin{tabular}{|c|c|l|l|l|l|l|}
\hline \multirow{2}{*}{ Sample } & \multicolumn{2}{|c|}{ Weight $(\mathrm{g})$} & \multicolumn{2}{c|}{ Color of sample } & \multicolumn{2}{c|}{ Form of sample } \\
\cline { 2 - 7 } & Initial & Final & Initial & Final & Initial & Final \\
\hline 1. & 1 & 1 & Transparent & Transparent & Chips & Chips \\
\hline 2. & 1 & 1 & Black & Black & Chips & Chips \\
\hline 3. & 1 & 1 & Pink & Pink & Chips & Chips \\
\hline 4. & 1 & 1 & White & White & Chips & Chips \\
\hline 5. & 1 & 1 & Orange & Orange & Chips & Chips \\
\hline 6. & 1 & 1 & Yellow & Yellow & Chips & Chips \\
\hline 7. & 1 & 1 & White & White & Chips & Chips \\
\hline 8. & 1 & 1 & Blue & Faded & Chips & $\begin{array}{l}\text { Layers detached } \\
\text { From each other }\end{array}$ \\
\hline 9. & 1 & 1 & Blue & Faded & Chips & Chips \\
\hline
\end{tabular}

The microbes, from forest soil and automobile wash-out sludge, were incubated in Burk's medium (without carbon source) with PEG and in the other set with plastic to observe the capability of the microbes to degrade PEG and plastic and a viability test was done. Viability test of the microbes from both the experiment i.e., inoculated with PEG and inoculated with different types of plastic shows that distinct colonies appear in all the plates. Moreover inoculation of microbes isolated from forest soil and automobile wash-out sludge in a medium containing plastic as carbon source was done and viability test showed that the microbes were alive in both the medium. After 45 days of incubation, the SEM pictures of different plastic samples indicate that there was no remarkable surface deformation except slight deformation on the surface of the soft drink glass. There were some cavities on the surface of this particular variety of plastic. The other variety of plastic does not show any remarkable change on their surface, except some microbes on the surface of the plastic.

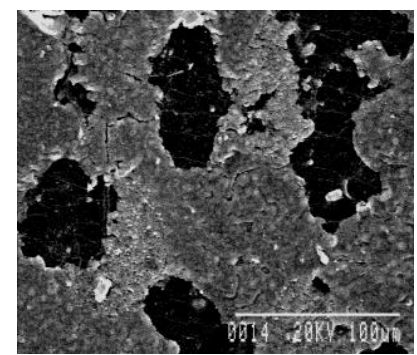

Fig.-1: Cavities on the surface of the plastic

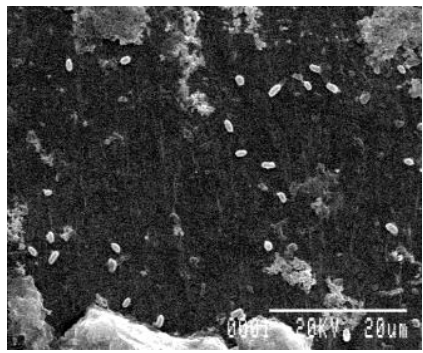

Fig.-2: Microbes on the surface of the plastic

\section{DISCUSSIONS}

The incubation of plastic sample under forest soil and automobile wash-out sludge for six month indicates that the microbes are not able to degrade the plastic in natural condition. Similar observation was recorded by Kathiresan, 2003 [8]. The result of incubation of microbes isolated from forest soil and automobile wash-out sludge in Burk's medium with PEG as carbon source indicates that microbes from both sources were alive in PEG medium [12]. Therefore it may be hypothesized that as there were no other carbon source rather than PEG, the microbes might get their required carbon by breaking the long carbon chain of PEG. After inoculation of the microbes for 30 days in Burk's medium (without sucrose) containing plastic as carbon source a viability test was done. From the viability test it was observed that microbes were alive in the medium containing only plastic as carbon source. In the SEM pictures some deformities were found in case of the plastic soft drink glass which showed a little irregularity in both the mediums (including soil microbes and sludge microbes). This particular variety of plastic is little hard in nature and showed little different surface character in microbiological test. There were some cavities on the surface of the plastic (Fig.1). Perhaps this is due to the presence of some heterogenic zones on the surface of the plastic. 
On the surface of other varieties of plastic there were some microbes (Fig.2) but it cannot be determined whether they are alive or not. Therefore it may be speculated that the microbes may have enough power to degrade plastic as they can degrade PEG because both PEG and plastic are polymer.

\section{CONCLUSIONS}

Plastic degradation in the forest soil indicates that there is no significant mass and surface deformation change after 180 days of inoculation. Similar unchanged result was recorded from the sample collected from auto mobile wash out sludge except variety- 8 in which it was observed that the different layers became detached from each other. Moreover viability test after 30 days of incubation and isolated microbes survive in the PEG medium showed that the microbes were alive in the said medium. SEM pictures also showed cavity like structures on the surface of the plastic and there were some microbes on the surface of the plastic. Now it can be hypothesized that these microbes may have enough power to degrade the plastic material with due course of time as both PEG and plastic are polymers. Therefore further study is needed to confirm the hypothesis.

\section{ACKNOWLEDGEMENT}

Authors are thankful to the forest officer of Ramana Bagan Forest, Burdwan, for giving permission to conduct the said experiment. Authors also like to extend their gratitude to all faculty members of Department of Environmental Science, The University of Burdwan for their active help and support.

\section{REFERENCE}

1. S. Kumar, A. A. M. Hatha, K. S. Christi, Diversity and effectiveness of tropical mangrove soil microflora on the degradation of polythene carry bags. Int. J. Trop. Biol. 55 (3-4), 2007, 777-786.

2. W.B. Bollag, J. Dec, J.M. Bollag, Biodegradation \& encylopedia of microbiology. In J.Lederberg (ed.). (Academic, New York 2000) 461-471.

3. M. Alexander, Biodegradation of chemicals of environmental concern. Sci. 211,1981, 132-138.

4. D. Y. Kim, H. Y. Rhee, Biodegradation of microbial and synthetic polyesters by fungi. Appl Microbiol Biotechnol. 61, 2003, 300-308.

5. G. Swift, Non-medical biodegradable polymers: Environmentally degradable polymers, In J. Domb, J. Kost, and D. M. Wiseman, eds. Handbook of Biodegradable Polymers, (Harwood Academic. Amsterdam, 1997) 473-511.

6. P. Sadocco, S. Nocerino, E. Dubini-Paglia, A. Seves, G. Elegir, Characterization of a poly (3-hydroxybutyrate) depolymerase from Aureobacterium saperdae: Active site and kinetics of hydrolysis studies, J Environ Poly Deg. 5,1997, 57-65.

7. T.N. Kambe, S. Y. Akutsu, N. Nomura, F. Onuma, T. Nakahara, Microbial degradation of polyurethane, polyester polyurethanes and polyether polyurethanes. Appl Microbiol Biotechnol. 51, 1999, 134 -140.

8. K. Kathiresan, Polythene and Plastics-degrading microbes from the mangrove soil, Rev. Biol. Trop. 51(3), 2003, 629-634.

9. G. L. Griffin, Biodegradable Fillers in Thermoplastic. Adv Chem Ser. 134, 1973, 159-162.

10. J.S. Webb, M. Nixon, I.M. Eastwood, M. Greenhalgh, G.D. Robson, P.S. Handley, Fungal colonization and biodeterioration of plasticized polyvinyl chloride, Appl Environ Microbiol. 66, 2000, 3194 -3200.

11. R. Premraj, M. Dobley, Biodegradation of Polymers, Ind. J. Biotechnol. 4, 2005, 186-193.

12. F. Kawai, S. Enokibara, Symbiotic degradation of polyethylene glycol (PEG) 20,000 phthalate polyester by phthalate ester- and PEG 20,000-utilizing bacteria, Appl. Environ Microbiol. 51(5),1996, 583-587. 